\title{
Laser phase noise to intensity noise conversion by lowest-order group-velocity dispersion in optical fiber: exact theory
}

\author{
W. K. Marshall, B. Crosignani, ${ }^{*}$ and A. Yariv \\ Department of Applied Physics, 128-95, California Institute of Technology, Pasadena, California 91125
}

Received September 10, 1999

\begin{abstract}
An exact result for the spectral density of intensity variations that occur after propagation of ergodic light in a medium having lowest-order-only group-velocity dispersion is obtained and applied to the problem of semiconductor laser phase noise to intensity noise conversion in a single-mode optical fiber. It is shown that the intensity spectrum after propagation formally approaches, for a large laser linewidth or a long (or highdispersion) fiber, the intensity spectrum of a thermal source having the same line shape as the laser. (C) 2000 Optical Society of America

OCIS codes: $\quad 060.2430,140.5960,260.2030,270.2500,060.5060$.
\end{abstract}

The conversion of optical phase variations to intensity variations by group-velocity dispersion (GVD) in optical fiber is a well-known phenomenon which occurs both for phase modulation and for phase noise. The effect of GVD in fiber on variations due to sinusoidal phase modulation can be modeled by expanding the time dependence of the phase-modulated input field in a Bessel-coefficient Fourier expansion. ${ }^{1}$ Phase noise can also be considered following such an approach, but only with the approximation that phase variations at a given frequency contribute solely to intensity variations at that same frequency - otherwise the calculation becomes intractable. ${ }^{2}$ Analyses valid in the limiting cases of small amplitude variations (i.e., a small-signal model ${ }^{3}$ ) and small propagation distance and low frequency ${ }^{4}$ have also been presented.

The small-signal model assumes that the optical field can be described as $E(t)=\sqrt{I_{0}}[1+\Delta(t)+$ $i \phi(t)] \exp \left(i \omega_{0} t\right)$, where $\Delta(t)$ and $\phi(t)$ are small, zeromean amplitude variations and phase variations, respectively. This leads to an expression for the relative intensity noise (RIN) after dispersive propagation of the form

$$
\begin{aligned}
\operatorname{RIN}(\Omega, z)= & 4\left[S_{\Delta \Delta}(\Omega) \cos ^{2} \theta-\operatorname{Re}_{\Delta \phi}(\Omega) \sin 2 \theta\right. \\
& \left.+S_{\phi \phi}(\Omega) \sin ^{2} \theta\right],
\end{aligned}
$$

where $\Omega$ is the angular frequency, $z$ is the propagation distance, and for lowest-order GVD, $\theta=-\beta_{0}^{\prime \prime} z \Omega^{2} / 2$. Here $S_{\Delta \Delta}(\Omega)$ is the spectral density of the normalized amplitude variations of the source, $S_{\phi \phi}(\Omega)$ is the spectral density of phase variations of the source, and $S_{\Delta \phi}(\Omega)$ is the cross-spectral density characterizing amplitude-phase correlations of the source.

The above-described model assumes that the total phase excursion is small, $|\phi(t)| \ll<1$. For a laser, whose phase exhibits a nonstationary random walk, this is true only over an interval on the order of the coherence time, $\tau_{\text {coh }}$. The small-propagation-distance model $^{4}$ does not require that phase variations be small but yields results that are valid only over a narrow range of (low) frequencies. Practically speaking, both these approaches can fail in cases of large field linewidths and in situations involving large-angle phase modulation. The former is the subject of this Letter; the latter, which involves nonergodic fields, will be addressed in a separate publication. ${ }^{5}$

In this Letter we present an exact theory for the spectral density of the intensity of ergodic (i.e., time average $=$ ensemble average) light after propagation in a medium exhibiting lowest-order-only GVD, a good approximation to standard single-mode optical fiber at $1550 \mathrm{~nm}$. We apply the theory to obtain a formula for the intensity noise spectrum that results, after propagation, from phase noise in light from a semiconductor laser. We also show that for any ergodic source the intensity spectrum formally approaches, for large values of the dispersion-distancefrequency product, that of a thermal source having an identical line shape.

The field envelope after propagation in a dispersive fiber is described by the solution to the optical field envelope equation,

$$
\frac{\partial \mathscr{E}}{\partial z}+\beta_{0}^{\prime} \frac{\partial \mathscr{E}}{\partial t}-\frac{i}{2} \beta_{0}^{\prime \prime} \frac{\partial^{2 \mathscr{E}}}{\partial t^{2}}=0
$$

where $\beta_{0}^{\prime}$ is the inverse of the group velocity at $\omega_{0}$ and $\beta_{0}^{\prime \prime}$ characterizes the lowest-order GVD. After absorbing the group delay $\beta_{0}^{\prime} z$ into the time variable, $t$, we obtain from Eq. (2)

$$
\mathscr{E}(t, z)=\left(\frac{i}{2 \pi\left|\beta_{0}^{\prime \prime} z\right|}\right)^{1 / 2} \int_{-\infty}^{\infty} \mathscr{E}_{0}(t+s) \exp \left(\frac{i s^{2}}{2 \beta_{0}^{\prime \prime} z}\right) \mathrm{d} s
$$

where $\mathscr{E}_{0}(t)$ is the field envelope at the fiber input and the remaining factors describe the field impulse response of the fiber. The optical intensity at $z$ is $I(t, z)=|\mathscr{E}(t, z)|^{2}$.

For an ergodic field, the Wiener-Khintchine theorem gives the one-sided spectral density of intensity as

$$
S_{I I}(\Omega, z)=2 \int_{-\infty}^{\infty}\langle I(0, z) I(\tau, z)\rangle \exp (-i \Omega \tau) \mathrm{d} \tau,
$$


where \langle\rangle indicates ensemble averaging. Equation (3) gives

$$
\begin{aligned}
& \langle I(0, z) I(\tau, z)\rangle=\frac{1}{4 \pi^{2} \beta_{0}^{\prime \prime 2} z^{2}} \\
& \quad \times \iiint \int\left\langle\mathscr{E}_{0}^{*}\left(s_{1}\right) \mathscr{E}_{0}\left(s_{2}\right) \mathscr{E}_{0}^{*}\left(\tau+s_{3}\right) \mathscr{E}_{0}\left(\tau+s_{4}\right)\right\rangle \\
& \quad \times \exp \left[\frac{i\left(-s_{1}^{2}+s_{2}^{2}-s_{3}^{2}+s_{4}^{2}\right)}{2 \beta_{0}^{\prime \prime} z}\right] \mathrm{d} s_{1} \mathrm{~d} s_{2} \mathrm{~d} s_{3} \mathrm{~d} s_{4},
\end{aligned}
$$

with limits $\pm \infty$ for each of the four integrations. Making the substitutions $s_{1} \rightarrow u_{1}, s_{2} \rightarrow u_{1}+u_{2}, s_{3} \rightarrow$ $u_{1}+u_{3}-\tau$, and $s_{4} \rightarrow u_{1}+u_{4}-\tau$, completing the $u_{1}$ integral [which yields factors $2 \pi\left|\beta_{0}^{\prime \prime} z\right| \delta\left(u_{2}-u_{3}+u_{4}\right)$ ], completing the $u_{3}$ integral, and then using Eq. (4), we obtain the following simple and useful expression for $S_{I I}(\Omega, z)$ :

$$
\begin{aligned}
S_{I I}(\Omega, z)= & 2 \int_{-\infty}^{\infty}\left\langle\mathscr{E}_{0}^{*}(0) \mathscr{E}_{0}\left(\beta_{0}^{\prime \prime} z \Omega\right) \mathscr{\mathscr { E }}_{0}^{*}\left(\beta_{0}^{\prime \prime} z \Omega+u\right) \mathscr{E}_{0}(u)\right\rangle \\
& \times \exp (-i \Omega u) \mathrm{d} u
\end{aligned}
$$

Equation (6), the first main result of this Letter, gives the spectral density of the intensity after propagation in linear, dispersive (lowest-order GVD only) fiber for any ergodic input field, $\mathscr{E}_{0}(t)$. A similar result which holds for non-ergodic fields will be described in Ref. 5 .

As a simple test of Eq. (6), we can first consider the properties of "thermal" light after propagation in fiber. Thermal light has uncorrelated real and imaginary field components that are identically zeromean Gaussian distributed. The Gaussian moment theorem ${ }^{6}$ in this case leads to

$$
\begin{aligned}
& \left\langle\mathscr{E}_{0}^{*}\left(t_{1}\right) \mathscr{E}_{0}\left(t_{2}\right) \mathscr{E}_{0}^{*}\left(t_{3}\right) \mathscr{E}_{0}\left(t_{4}\right)\right\rangle=\left\langle\mathscr{E}_{0}^{*}\left(t_{1}\right) \mathscr{E}_{0}\left(t_{2}\right)\right\rangle \\
& \quad \times\left\langle\mathscr{E}_{0}^{*}\left(t_{3}\right) \mathscr{E}_{0}\left(t_{4}\right)\right\rangle+\left\langle\mathscr{E}_{0}^{*}\left(t_{1}\right) \mathscr{E}_{0}\left(t_{4}\right)\right\rangle\left\langle\mathscr{E}_{0}^{*}\left(t_{3}\right) \mathscr{E}_{0}\left(t_{2}\right)\right\rangle .
\end{aligned}
$$

For thermal light, Eq. (6) therefore reduces to

$$
\begin{aligned}
S_{I I}(\Omega, z)= & 2 \int_{-\infty}^{\infty}\left\{\left|\left\langle\mathscr{E}_{0}^{*}(0) \mathscr{E}_{0}\left(\beta_{0}^{\prime \prime} z \Omega\right)\right\rangle\right|^{2}\right. \\
& \left.+\left|\left\langle\mathscr{E}_{0}^{*}(0) \mathscr{E}_{0}(u)\right\rangle\right|^{2}\right\} \exp (-i \Omega u) \mathrm{d} u \\
= & 4 \pi\left\langle\left|\mathscr{E}_{0}^{*}(t)\right|^{2}\right\rangle^{2} \delta(\Omega) \\
& +\frac{1}{4 \pi} \int_{-\infty}^{\infty} S_{\mathscr{E}_{0}^{*} \mathscr{E}_{0}}(\omega) S_{\mathscr{E}_{0}^{*} \mathscr{E}_{0}}(\omega-\Omega) \mathrm{d} \omega,
\end{aligned}
$$

where $S_{\mathscr{E}_{0}^{* *} \mathscr{E}_{0}}(\omega)=2 \int_{-\infty}^{\infty}\left\langle\mathscr{E}_{0}^{*}(0) \mathscr{E}_{0}(\tau)\right\rangle \exp (-i \omega \tau) \mathrm{d} \tau$ is the spectral density of the input field envelope. For thermal light, $S_{I I}(\Omega, z)$ is (aside from a delta function representing the $\mathrm{cw}$ intensity) the convolution of $S_{\mathscr{E}_{0}^{*} \mathscr{E}_{0}}(\omega)$ with $S_{\mathscr{E}_{0}^{*} \mathscr{E}_{0}}(-\omega)$, independent of $z$.

We now consider laser phase noise. The phase of a laser is nonstationary and has a (time-varying) Gaussian distribution, whereas the laser electric field envelope is ergodic but non-Gaussian. Taking $\mathscr{E}_{0}(t)=$ $\sqrt{I_{0}} \exp [i \phi(t)]$ (that is, neglecting the quenched am- plitude fluctuations of the laser), defining $\psi\left(t_{1}, t_{2}\right) \equiv$ $\phi\left(t_{2}\right)-\phi\left(t_{1}\right)$, and following steps commonly used in calculating the laser line shape, one obtains

$$
\begin{aligned}
\left\langle\mathscr{E}_{0}^{*}\left(t_{1}\right) \mathscr{E}_{0}\left(t_{2}\right)\right\rangle & =I_{0}\left\langle\exp \left[i \psi\left(t_{1}, t_{2}\right)^{2}\right]\right\rangle \\
& =I_{0} \exp \left(-\left\langle\psi\left(t_{1}, t_{2}\right)^{2}\right\rangle / 2\right) .
\end{aligned}
$$

Taking advantage of the algebraic identity

$$
\left[\psi\left(t_{1}, t_{2}\right)+\psi\left(t_{3}, t_{4}\right)\right]^{2}=\sum_{p=1}^{3} \sum_{q=p}^{3}(-1)^{p+q} \psi\left(t_{p}, t_{q+1}\right)^{2}
$$

gives, by a similar calculation,

$$
\begin{aligned}
\left\langle\mathscr{E}_{0}^{*}\left(t_{1}\right) \mathscr{E}_{0}\left(t_{2}\right) \mathscr{E}_{0}^{*}\left(t_{3}\right) \mathscr{E}_{0}\left(t_{4}\right)\right\rangle=I_{0}^{2} \prod_{p=1}^{3} \prod_{q=p}^{3} \\
\times \exp \left[\frac{(-1)^{p+q+1}}{2}\left\langle\psi\left(t_{p}, t_{q+1}\right)^{2}\right\rangle\right] .
\end{aligned}
$$

Equation (6) then gives, for the case of Gaussian phase noise,

$$
\begin{aligned}
& \operatorname{RIN}(\Omega, z)=2 \exp \left(-\left\langle\psi\left(0, \beta_{0}^{\prime \prime} z \Omega\right)^{2}\right\rangle\right) \int_{-\infty}^{\infty} \exp (-i \Omega u) \\
& \quad \times\left\{\operatorname { e x p } \left[\frac{\left\langle\psi\left(0, u-\beta_{0}^{\prime \prime} z \Omega\right)^{2}\right\rangle}{2}+\frac{\left\langle\psi\left(0, u+\beta_{0}^{\prime \prime} z \Omega\right)^{2}\right\rangle}{2}\right.\right. \\
& \left.\left.\quad-\left\langle\psi(0, u)^{2}\right\rangle\right]-1\right\} \mathrm{d} u
\end{aligned}
$$

where $\operatorname{RIN}(\Omega, z)$, the relative intensity noise, is related to $S_{I I}(\Omega, z)$ by $\operatorname{RIN}(\Omega, z)=\left[S_{I I}(\Omega, z) /\langle I\rangle^{2}\right]-4 \pi \delta(\Omega)$. Equation (12), the second main result of this Letter, gives the spectral density of normalized intensity variations in laser light after propagation in dispersive fiber, under the approximations that higher-order dispersion and any effect of optical nonlinearity in the fiber can be neglected and that the effect of laser phase noise which is converted to amplitude noise dominates the effect of any residual laser amplitude noise.

Equation (12) can be evaluated numerically, if desired, for any particular $\left\langle\psi(0, t)^{2}\right\rangle$ (which will depend on the dynamics of the laser). However, of more interest here is the fact that Eq. (12) can be evaluated analytically in the case in which the frequencyfluctutation spectrum of the laser is approximately white [i.e., $S_{\omega \omega}(\Omega)=2 \Delta \omega$, where $\Delta \omega$ is the FWHM laser linewidth]. In this case, $\left\langle\psi(0, t)^{2}\right\rangle=\Delta \omega|t|$ and Eq. (12) leads to the explicit result that

$$
\begin{aligned}
\operatorname{RIN}(\Omega, z) & =\frac{4 \Delta \omega}{\Delta \omega^{2}+\Omega^{2}}\left\{1-\exp \left(-\Delta \omega\left|\beta_{0}^{\prime \prime} z \Omega\right|\right)\right. \\
\times & {\left.\left[\cos \left(\Omega\left|\beta_{0}^{\prime \prime} z \Omega\right|\right)+\frac{\Delta \omega}{\Omega} \sin \left(\Omega\left|\beta_{0}^{\prime \prime} z \Omega\right|\right)\right]\right\} . }
\end{aligned}
$$




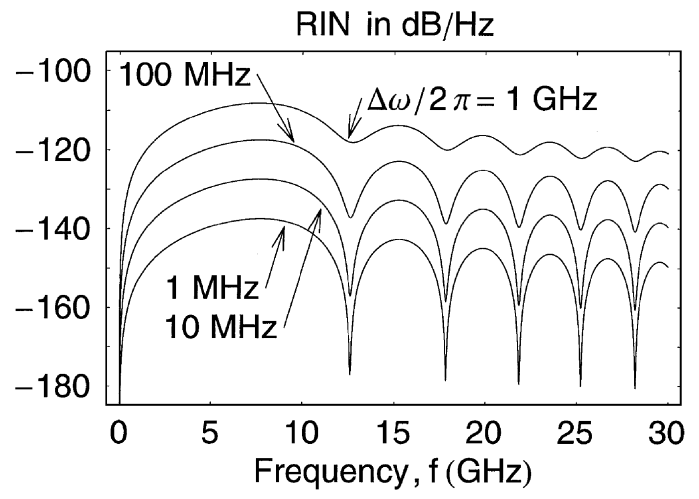

Fig. 1. RIN from Eq. (13) plotted versus frequency, $f=\Omega /(2 \pi)$, for linewidths $\Delta \omega /(2 \pi)=1 \mathrm{GHz}, 100 \mathrm{MHz}$, $10 \mathrm{MHz}, 1 \mathrm{MHz}$ and $\beta_{0}^{\prime \prime} z=1000 \mathrm{ps}^{2}$ (equivalent to $\sim 50 \mathrm{~km}$ of standard single-mode fiber at $1550 \mathrm{~nm}$ ).

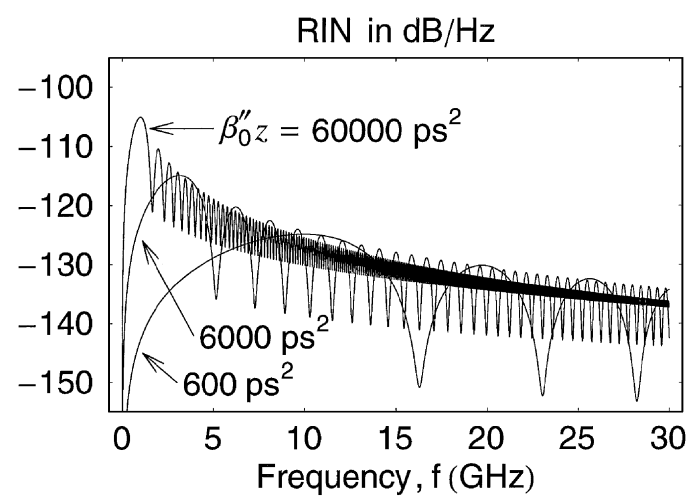

Fig. 2. RIN from Eq. (13) plotted versus frequency, $f=$ $\Omega /(2 \pi)$, for $\beta_{0}^{\prime \prime} z=60000,6000,600 \mathrm{ps}^{2}$ and linewidth $\Delta \omega /(2 \pi)=30 \mathrm{MHz}$. The equivalent distances in standard single-mode fiber would be $z \simeq 30,300,3000 \mathrm{~km}$.

Equation (13), the third main result of this Letter, is plotted in Figs. 1 and 2.

For $\Omega \ll\left|\beta_{0}^{\prime \prime} z \Delta \omega\right|^{-1}$, Eq. (13) reduces ${ }^{7}$ to

$$
\operatorname{RIN}(\Omega, z) \simeq \frac{8 \Delta \omega}{\Omega^{2}} \sin ^{2}\left(\frac{1}{2} \beta_{0}^{\prime \prime} z \Omega^{2}\right),
$$

which is exactly the small-signal result given by the last term only (i.e., neglecting laser amplitude variations) on the right-hand side of Eq. (1). For $\Omega \gg$ $\left|\beta_{0}^{\prime \prime} z \Delta \omega\right|^{-1}$, Eq. (13) instead leads to

$$
\operatorname{RIN}(\Omega, z) \simeq \frac{4 \Delta \omega}{\Delta \omega^{2}+\Omega^{2}},
$$

i.e., to a Lorentzian with FWHM of $2 \Delta \omega$, twice that of the laser field spectrum.

With the above-reported results, we are in a position to evaluate the influence of large phase variations on the process of phase noise to amplitude noise conversion in fiber. The effects of large phase variations are twofold: First, a large phase variation at a given frequency $\Omega$ (or within a narrow range of $\Omega$ ) causes an amplitude variation after propagation which is of a different relative (i.e., nonproportional) magnitude than that caused by a small phase variation, in the same way that the large-signal amplitude response to phase modulation is different from the small-signal response. Second, a large phase variation at $\Omega$ can lead to significant amplitude variations after propagation not just at $\Omega$ but also at multiples of $\Omega$. A comparison of the results for phase noise in Eqs. (15) and (19) of Ref. 2 with the results for phase modulation given in Ref. 1 reveals that only the first of these two effects is included in the results of Ref. 2.

A result comparable to Eq. (15) can be seen to hold for any ergodic input field with a finite coherence time, since Eq. (6) can be rewritten as

$$
\begin{aligned}
S_{I I}(\Omega, z)= & 2 \int_{-\infty}^{\infty} \exp (-i \Omega u)\left\langle\left[\mathscr{E}_{0}^{*}(0) \mathscr{E}_{0}(u)\right.\right. \\
& \left.-\left\langle\mathscr{E}_{0}^{*}(0) \mathscr{E}_{0}(u)\right\rangle\right]\left[\mathscr{E}_{0}^{*}\left(\beta_{0}^{\prime \prime} z \Omega+u\right)\right. \\
& \left.\left.\times \mathscr{E}_{0}\left(\beta_{0}^{\prime \prime} z \Omega\right)-\left\langle\mathscr{E}_{0}^{*}(u) \mathscr{E}_{0}(0)\right\rangle\right]\right\rangle \mathrm{d} u \\
& +2 \int_{-\infty}^{\infty}\left|\left\langle\mathscr{E}_{0}^{*}(0) \mathscr{E}_{0}(u)\right\rangle\right|^{2} \exp (-i \Omega u) \mathrm{d} u .
\end{aligned}
$$

For $\left|\beta_{0}^{\prime \prime} z \Omega\right| \gg \tau_{\text {coh }}$, the variations in $\mathscr{E}_{0}^{*}(0) \mathscr{E}_{0}(u)$ from its mean, $\left\langle\mathscr{E}_{0}^{*}(0)^{\mathscr{E}} \mathscr{E}_{0}(u)\right\rangle$, will approach zero correlation with variations in $\mathscr{E}_{0}^{*}\left(\beta_{0}^{\prime \prime} z \Omega+u\right)^{\mathscr{E}_{0}}\left(\beta_{0}^{\prime \prime} z \Omega\right)$ from its mean, $\left\langle\mathscr{E}_{0}^{*}(u)^{\mathscr{E}} \mathscr{E}_{0}(0)\right\rangle$. In this case the first term on the right-hand side of Eq. (16) will approach zero, so that $S_{I I}(\Omega, z) \rightarrow 2 \int_{-\infty}^{\infty}\left|\left\langle\mathscr{E}_{0}^{*}(0) \mathscr{E}_{0}(u)\right\rangle\right|^{2} \exp (-i \Omega u) \mathrm{d} u$. Thus, the intensity noise spectrum after dispersive propagation approaches (albeit nonuniformly in $\Omega$ ) that of a thermal source [Eq. (8)] having the same initial field spectral density.

This work was supported by the U.S. Office of Naval Research, the Defense Advanced Research Projects Agency, and the U.S. Air Force Office of Scientific Research under grant N00014-91-J-1195.

*Permanent address, Dipartimento di Fisica, Università dell'Aquila, L'Aquila, Italy, and Instituto Nazionale Fisica della Materia, Unit a' dell'Università di Roma "La Sapienza," Roma L'Aquila, Italy.

\section{References}

1. A. Chraplyvy, R. Tkach, L. Buhl, and R. Alferness, Electron. Lett. 22, 409 (1986).

2. S. Yamamoto, N. Edagawa, H. Taga, Y. Yoshida, and H. Wakabayashi, J. Lightwave Technol. 8, 1716 (1990).

3. J. Wang and K. Petermann, J. Lightwave Technol. 10, 96 (1992).

4. K. Petermann, Electron. Lett. 26, 2097 (1990).

5. W. K. Marshall and A. Yariv, "Spectrum of the intensity of modulated noisy light after propagation in dispersive fiber," IEEE Photon. Technol. Lett. (to be published).

6. J. W. Goodman, Statistical Optics (Wiley, New York, 1985).

7. It is useful to note that $\left|\beta_{0}^{\prime \prime} z \Omega \Delta \omega\right| \ll 1$ implies that either $\left|\beta_{0}^{\prime \prime} z \Omega^{2}\right| \ll 1$ or $\Delta \omega / \Omega \ll 1$. 